\title{
Deletion of $(26,6)$-Simple Points as Multivalued Retractions
}

\author{
Carmen Escribano, Antonio Giraldo, and María Asunción Sastre* \\ Departamento de Matemática Aplicada, Facultad de Informática \\ Universidad Politécnica, Campus de Montegancedo \\ Boadilla del Monte, 28660 Madrid, Spain \\ \{cescribano, agiraldo, masastre\}@fi.upm.es
}

\begin{abstract}
In a recent paper we have introduced a notion of multivalued continuity in digital spaces which extends the usual notion of digital continuity and allows to define topological notions, like retractions, in a far more realistic way than by using just single-valued digitally continuous functions. In particular, we have characterized the deletion of simple points in 2-D, one of the most important processing operations in digital topology, as a particular kind of retraction. In this work we extend some of these results to 3 -dimensional digital sets.
\end{abstract}

Keywords: Digital topology, continuous multivalued function, simple point, retraction.

\section{Introduction}

Digitally continuous maps were first introduced by A. Rosenfeld [15] in 1986. He characterized them as functions taking connected sets to connected sets.

Results related with this type of continuity were proved also by L. Boxer [2 4], who introduced such notions as digital homeomorphism, retracts and homotopies. A different approach using multivalued maps was suggested by V. Kovalevsky [13]. (See the introduction of [5] for a discussion of the limitations of these and related approaches).

In recent papers [5, 6], the authors presented a theory of continuity in digital spaces which extends the one introduced by Rosenfeld. Our approach uses multivalued maps and provides a better framework to define topological notions, like retractions, in a far more realistic way than by using just single-valued digitally continuous functions. This notion has allowed us to characterize most common thinning algorithms for digital images as retractions.

In this work we extend some results of those papers to 3-dimensional digital sets. In sections 2 and 3 we revise the basic notions on digital topology required throughout the paper. In section 4 we recall the notions of a digitally continuous multivalued function and of a digital retraction, formulating one of the results of

\footnotetext{
* The authors have been supported by MICINN MTM2009-07030 (A.Giraldo) and UPM-2011-Q061010133.
} 
[5] for 3-dimensional sets (Proposition 2). In section 5 we prove the main result of the paper (Theorem 3 ): If a point $p$ is $(26,6)$-simple in $X$, then there exists a multivalued $(\mathcal{N}, 26)$-retraction $F: X \longrightarrow X \backslash\{p\}$.

For information on Digital Topology we recommend the survey [11] and the books by Kong and Rosenfeld [12], and by Klette and Rosenfeld [9].

We are grateful to the referees for helpful comments and suggestions.

\section{Digital Spaces}

Two points in the digital plane $\mathbb{Z}^{2}$ are 8 -adjacent if they are different and their coordinates differ in at most a unit. They are 4-adjacent if they are 8-adjacent and differ in at most a coordinate. Given $p \in \mathbb{Z}^{2}$ we define $\mathcal{N}(p)$ as the set of points 8-adjacent to $p$, i.e. $\mathcal{N}(p)=\left\{p_{1}, p_{2}, \ldots, p_{8}\right\}$. This is also denoted as $\mathcal{N}_{8}(p)$. Analogously, $\mathcal{N}_{4}(p)$ is the set of points 4 -adjacent to $p$ (with the above notation $\left.\mathcal{N}_{4}(p)=\left\{p_{2}, p_{4}, p_{6}, p_{8}\right\}\right)$.

Two points of the digital 3 -space $\mathbb{Z}^{3}$ are 26 -adjacent if they are different and their coordinates differ in at most a unit. They are called 18-adjacent if they are 26-adjacent and differ in at most two coordinates, and they are called 6-adjacent if they are 26-adjacent and differ in exactly one coordinate. We have therefore, three different neighborhoods of $p: \mathcal{N}_{26}(p)=\mathcal{N}(p), \mathcal{N}_{18}(p)$ and $\mathcal{N}_{6}(p)$.

In an analogous way, adjacency relations are defined in $\mathbb{Z}^{n}$ for $n \geq 4$.

A $k$-path $P$ in $\mathbb{Z}^{n}$ from the point $q_{0}$ to the point $q_{r}$ is a sequence $P=$ $\left\{q_{0}, q_{1}, q_{2}, \ldots, q_{r}\right\}$ of points such that $q_{i}$ is $k$-adjacent to $q_{i+1}$, for every $i \in$ $\{0,1,2, \ldots, r-1\}$. If $q_{0}=q_{r}$ then it is called a closed path. A set $S \subset \mathbb{Z}^{n}$ is $k$-connected if for every pair of points of $S$ there exists a $k$-path contained in $S$ joining them. A $k$-connected component of $S$ is a $k$-connected maximal set.

Given $X \subset \mathbb{Z}^{n}, p \in X$, we say, according to [12], that $p$ is a $k$-boundary point of $X$ if $\mathcal{N}_{\bar{k}}(p) \cap\left(\mathbb{Z}^{n} \backslash X\right) \neq \emptyset$, where $(k, \bar{k})=(8,4)$ if $n=2,(k, \bar{k})=(26,6)$ if $n=3$ (this notation will be used throughout the paper). We denote by $\partial_{k} X$ the set of $k$-boundary points of $X$.

A point $p$ and a set $X$ are $k$-adjacent if $p \notin X$ and there exists $q \in X$ such that $p$ and $q$ are $k$-adjacent.

\section{Digitally Continuous Single-Valued Functions}

We start this section revising the notion of digitally continuous function and some of its properties.

Definition 1. Let $f: X \subset \mathbb{Z}^{m} \longrightarrow \mathbb{Z}^{n}$ be a function between digital spaces with adjacency relations $k$ and $k^{\prime}$, respectively. According to [15] and [3], $f$ is $\left(k, k^{\prime}\right)$ continuous, if and only if, for every $p, p^{\prime} \in X k$-adjacent points of $\mathbb{Z}^{m}$ then either $f(p)=f\left(p^{\prime}\right)$ or $f(p)$ and $f\left(p^{\prime}\right)$ are $k^{\prime}$-adjacent points of $\mathbb{Z}^{n}$. When $m=n$ and $k=k^{\prime}, f$ is said to be just $k$-continuous. 
In [15] several results about digitally continuous functions related to operations with continuous functions, intermediate values property, almost-fixed point theorem, Lipschitz conditions, one-to-oneness, etc, were proved. Boxer [2-4] expanded this notion to digital homeomorphisms, retractions, extensions, homotopies, digital fundamental group, induced homomorphisms, etc. (see also [8] and [10] for previous related results).

In particular, Boxer proved that the $k$-boundary $\partial S$ of a digital square $S$ is not a digital $k$-retract of the square [2], i.e., it is not possible to construct a digitally continuous function $f: S \longrightarrow \partial S$ such that $f(x)=x$ for every $p \in \partial S$, as happens if we consider them as subsets of $\mathbb{R}^{2}$. However, neither the outer $k$-boundary of an annulus is a $k$-retract of the annulus, as opposite with what happens considering as subsets of $\mathbb{R}^{2}$. Therefore, digitally $k$-continuous single-valued functions can not model correctly the topology of $\mathbb{R}^{2}$. In the next section we show how it is possible to define a notion of continuity for multivalued functions in such a way that these limitations of digitally continuous single valued functions are alleviated (see Proposition 2).

\section{Digitally Continuous Multivalued Functions}

The definitions and results in this section were first introduced in [5].

Definition 2. The r-th subdivision of $\mathbb{Z}^{n}$ is formed by the set

$$
\mathbb{Z}_{r}^{n}:=\left\{\left(\frac{z_{1}}{3^{r}}, \frac{z_{2}}{3^{r}}, \ldots, \frac{z_{n}}{3^{r}}\right) \mid\left(z_{1}, z_{2}, \ldots, z_{n}\right) \in \mathbb{Z}^{n}\right\}
$$

and the function $i_{r}: \mathbb{Z}_{r}^{n} \longrightarrow \mathbb{Z}^{n}$ given by $i_{r}\left(\frac{z_{1}}{3^{r}}, \frac{z_{2}}{3^{r}}, \ldots, \frac{z_{n}}{3^{r}}\right):=\left(z_{1}^{\prime}, z_{2}^{\prime}, \ldots, z_{n}^{\prime}\right)$ where $\left(z_{1}^{\prime}, z_{2}^{\prime}, \ldots, z_{n}^{\prime}\right)$ is the point in $\mathbb{Z}^{n}$ which is closest to $\left(\frac{z_{1}}{3^{r}}, \frac{z_{2}}{3^{r}}, \ldots, \frac{z_{n}}{3^{r}}\right)$ in the Euclidean metric.

If we consider in $\mathbb{Z}^{n}$ a $k$-adjacency relation, we can consider in $\mathbb{Z}_{r}^{n}$ the induced adjacency relation, i.e., $\left(\frac{z_{1}}{3^{r}}, \frac{z_{2}}{3^{r}}, \ldots, \frac{z_{n}}{3^{r}}\right)$ and $\left(\frac{z_{1}^{\prime}}{3^{r}}, \frac{z_{2}^{\prime}}{3^{r}}, \ldots, \frac{z_{n}^{\prime}}{3^{r}}\right)$ are $k$-adjacent if and only if $\left(z_{1}, z_{2}, \ldots, z_{n}\right)$ is $k$-adjacent to $\left(z_{1}^{\prime}, z_{2}^{\prime}, \ldots, z_{n}^{\prime}\right)$.

Proposition 1. $i_{r}$ is $k$-continuous as a function between digital spaces.

Definition 3. Given $X \subset \mathbb{Z}^{n}$, the $r$-th subdivision of $X$ is the set $X_{r}:=i_{r}^{-1}(X)$.

Intuitively, if we consider $X$ made of pixels, (respectively, voxels), the $r$-th subdivision of $X$ consists in replacing each pixel with $9^{r}$ pixels (respectively, $27^{r}$ voxels) and the function $i_{r}$ is like an inclusion in the geometric sense.

Definition 4. Consider $X, Y \subset \mathbb{Z}^{n}$. A multivalued function $F: X \longrightarrow Y$ is a function $F$ such that for every $x \in X, F(x)$ is a non-empty subset of $Y$. A multivalued function $F: X \longrightarrow Y$ is said to be a $\left(k, k^{\prime}\right)$-continuous multivalued 
function if there exists a $\left(k, k^{\prime}\right)$-continuous (single-valued) function from $X_{r}$ to $Y$ for some $r \in \mathbb{N}$ such that $F(x):=\cup_{x^{\prime} \in i_{r}^{-1}(x)} f\left(x^{\prime}\right)$. We say then that $F$ is induced by $f$. When $k=k^{\prime}, F$ is said to be just a $k$-continuous multivalued function.

The reader is referred to [5] and [6] for properties of digitally continuous multivalued functions. We just note here that any single-valued digitally continuous function is continuous as a multivalued function, and that if $F$ is a $\left(k, k^{\prime}\right)$ continuous multivalued function then $F$ takes $k$-connected sets to $k^{\prime}$-connected sets.

Definition 5. Let $X \subset \mathbb{Z}^{n}$ and $Y \subset X$. We say that $Y$ is a multivalued $k$ retract of $X$ if there exists a $k$-continuous multivalued function $F: X \longrightarrow Y(a$ multivalued $k$-retraction) such that $F(y)=\{y\}$ if $y \in Y$. If moreover $F(x) \subset$ $\mathcal{N}(x)$ for every $x \in X \backslash Y$, we say that $F$ is a multivalued $(\mathcal{N}, k)$-retraction.

The following result, which generalizes a 2-dimensional result proved in [5], and that can be proved in a similar way as that result, shows that digitally continuous multivalued functions and, in particular, multivalued $k$-retractions and multivalued $(\mathcal{N}, k)$-retractions have similar properties as retractions in $\mathbb{R}^{3}$, in contrast with single-valued digital retractions, which, as noted in the introduction, have serious limitations to replicate the behavior of retractions in $\mathbb{R}^{3}$.

Proposition 2. The following holds:

i) The $k$-boundary of a cube $X$ (with Int $X \neq \emptyset$ ) is not a multivalued $k$-retract of $X$.

ii) The outer $k$-boundary $\partial_{k} X$ of a hollow cube $X$ is a multivalued $(\mathcal{N}, k)$ retract of $X$.

\section{Deletion of Simple Points as $(\mathcal{N}, k)$-Retractions}

A point $p \in X \subset \mathbb{Z}^{2}$ is 8-simple in $X$ if its deletion does not change the topology of $X$ in the sense that, when deleting it, the number of components and holes are preserved. A point $p \in X \subset \mathbb{Z}^{3}$ is 26-simple in $X$ if, after deleting it, the number and location of components, holes (tunnels) and cavities are preserved (see [1, 14] for the formal definition of these intuitive notions, and [7] for an example of why in the 3-dimensional case it is not enough to preserve just the number of holes).

A $k$-simple point can be locally detected by the following characterization:

Theorem $1\left([\mathbf{1},[\mathbf{1 4}])\right.$. Let $X \subset \mathbb{Z}^{2}$. A point $p \in X$ is 8-simple if and only if $p$ is an 8-boundary point of $X$ such that the number of 8-connected components of $\mathcal{N}(p) \cap X$ which are 8-adjacent to $p$ is equal to 1 .

Let $X \subset \mathbb{Z}^{3}$. A point $p \in X$ is 26-simple if and only if the number of 26connected components of $\mathcal{N}(p) \cap X$ which are 26-adjacent to $p$ is equal to 1 , and the number of 6 -connected components of $\mathcal{N}_{18}(p) \cap\left(\mathbb{Z}^{3} \backslash X\right)$ which are 6 -adjacent to $p$ is equal to 1 .

The following theorem was proved in [5]. 
Theorem 2. Consider $X \subset \mathbb{Z}^{2}$ and $p \in X$. Then $p$ is an 8-simple point if and only if there exists a multivalued $(\mathcal{N}, 8)$-retraction $F: X \longrightarrow X \backslash\{p\}$.

Theorem 2 also holds for $(k, \bar{k})=(4,8)$ with an additional condition on $p$.

The following result generalizes to $\mathbb{Z}^{3}$ the "only if" part of Theorem 2 .

Theorem 3. Consider $X \subset \mathbb{Z}^{3}$ and $p \in X$ a 26-simple point. Then there exists an $(\mathcal{N}, 26)$-retraction $F: X \longrightarrow X \backslash\{p\}$.

Proof. We show how to define a multivalued $(\mathcal{N}, 26)$-retraction $F: X \longrightarrow X \backslash\{p\}$ according to the number of points of $\mathcal{N}_{6}(p) \cap X$.

Denote the points in $\mathcal{N}_{6}(p)$ as follows: $n$ the point above $p, s$ the point below $p, w$ the point left of $p, e$ the point right of $p, f$ the point front of $p, b$ the point behind $p$. These 6 directions allows us to determine each point of $\mathcal{N}_{26}(p)$.

Case 1: $\operatorname{card}\left(\mathcal{N}_{6}(p) \cap X\right) \geq 3$. If $\operatorname{card}\left(\mathcal{N}_{6}(p) \cap X\right)=6, p$ is not 26-simple. Then $\mathcal{N}(p)$ is 26 -simple if and only if $p$ is, up to symmetries, as in Figure 1 , where the points not explicitly drawn can be in $X$ or in $\mathbb{Z}^{3} \backslash X$.
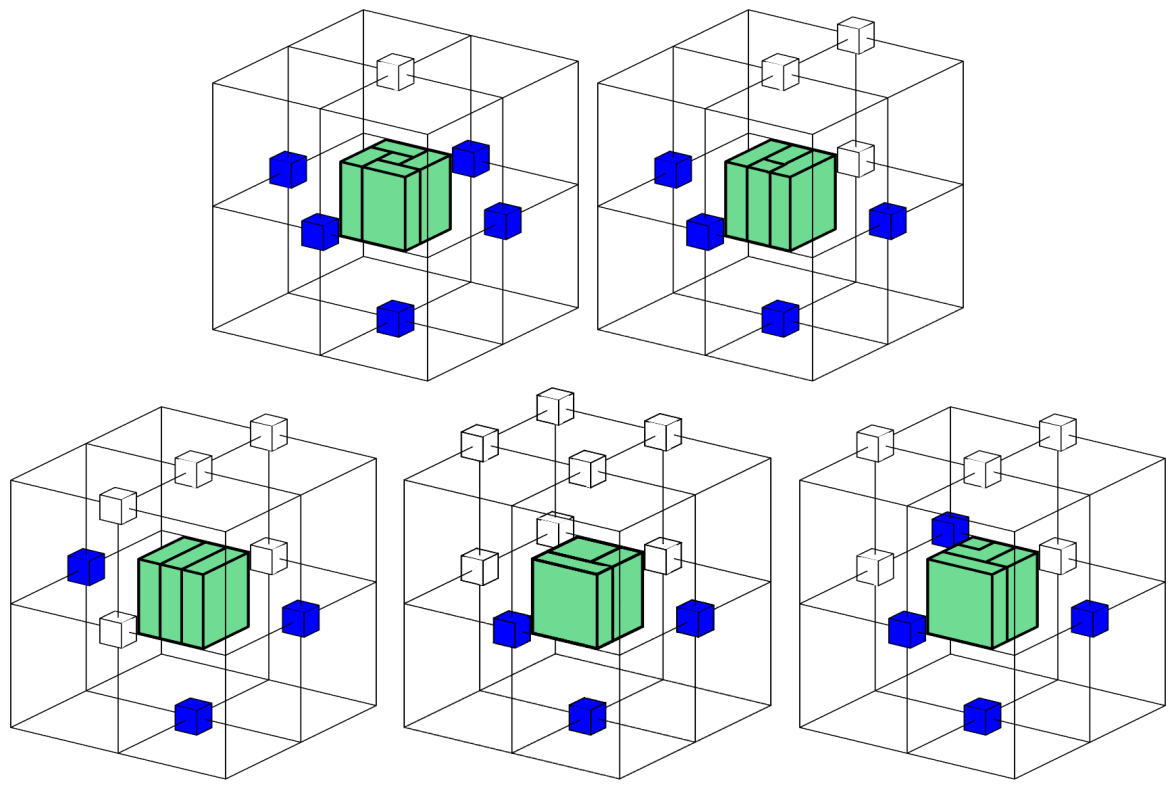

Fig. 1. Local configurations of a 26 -simple point with $\operatorname{card}\left(\mathcal{N}_{6}(p) \cap X\right) \geq 3$

If $\operatorname{card}\left(\mathcal{N}_{6}(p) \cap X\right)=5$, we subdivide $p$ in five parts $\left(A_{1}, A_{2}, A_{3}, A_{4}, A_{5}\right)$ as in Figure 1a and we define $f$ inducing $F$ such that $f\left(A_{i}\right)$ is the point of $\mathcal{N}_{6}(p) \cap X$ closest to $A_{i}$. Note that $f$ is continuous because if $A_{i}$ and $A_{j}$ are 26-adjacent then so are $f\left(A_{i}\right)$ and $f\left(A_{j}\right)$, and if a voxel in $\mathcal{N}(p)$ is 26-adjacent to $A_{i}$, then it is also 26 -adjacent to $f\left(A_{i}\right)$. 
If $\operatorname{card}\left(\mathcal{N}_{6}(p) \cap X\right)=4, \mathcal{N}(p)$ is, up to symmetries, as in Figure 1b. Since $p$ is simple, the point $n b$ (north and behind) must be in $\mathbb{Z}^{2} \backslash X$ because it is necessary to 6 -connect in $\mathcal{N}_{18}(p)$ the points $n$ and $b$ (see Theorem 1 ). To define $F$ we subdivide $p$ in 4 parts $\left(A_{1}, A_{2}, A_{3}, A_{4}\right)$ and define $f$ inducing $F$ such that $f\left(A_{i}\right)$ is the point of $\mathcal{N}_{6}(p) \cap X$ closest to $A_{i}$.

If $\operatorname{card}\left(\mathcal{N}_{6}(p) \cap X\right)=3$, then $p$ is, up to symmetries, as in one of the configurations of Figures 1c-1e (the configurations correspond to the different ways to 6 -connect in $\mathcal{N}_{18}(p)$ the 3 white points of $\left.\mathcal{N}_{6}(p)\right)$. In all cases we subdivide $p$ as in the figure and we define $f$ inducing $F$ such that $f\left(A_{i}\right)$ is the point of $\mathcal{N}_{6}(p) \cap X$ closest to $A_{i}$.
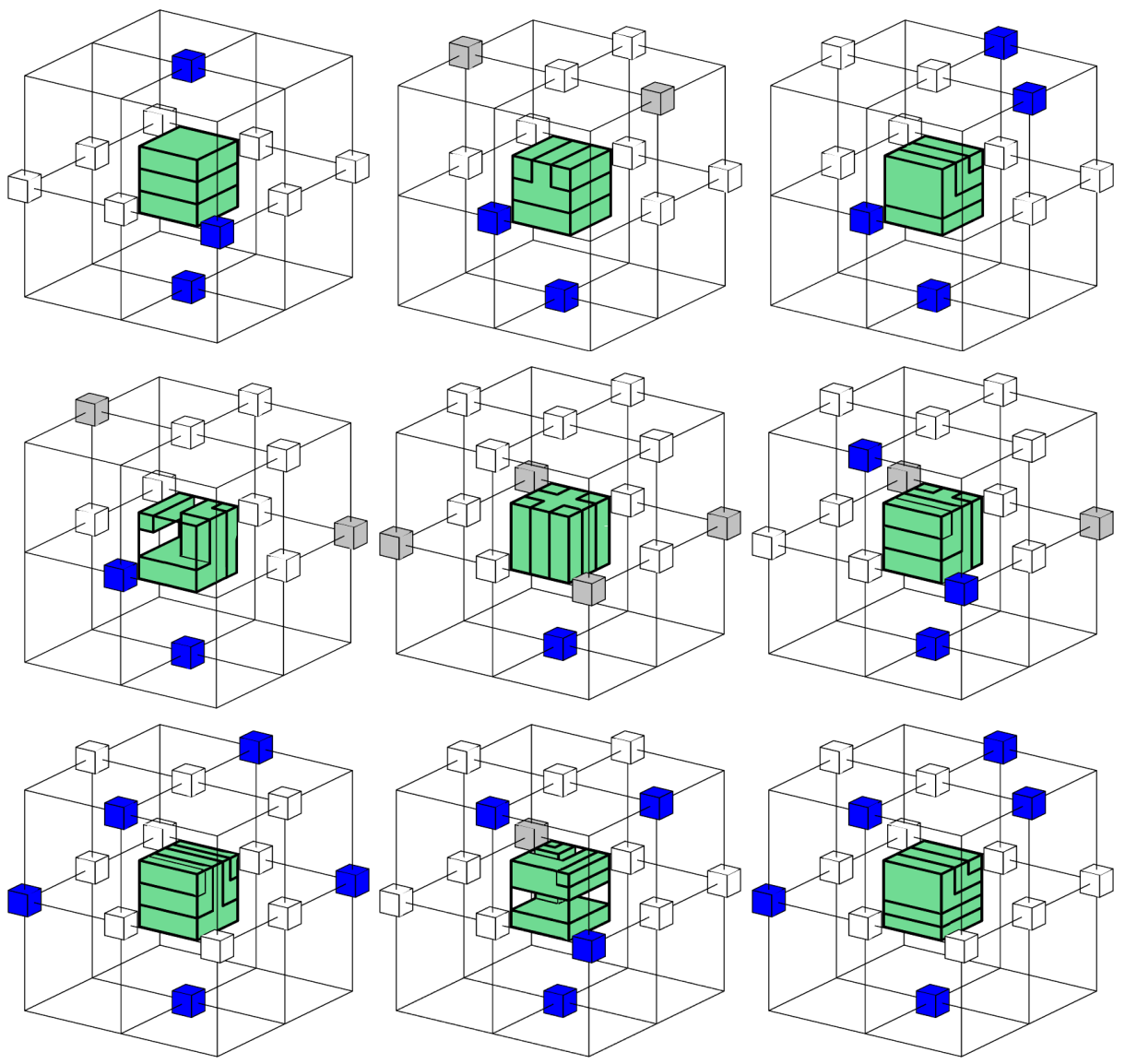

Fig. 2. Local configurations of a 26-simple point with $2 \geq \operatorname{card}\left(\mathcal{N}_{6}(p) \cap X\right) \geq 1$. There are three more configurations, interchanging $f e$ and $f w$ in each of the three last configurations (in each case the same subdivisions are used to define $F$ ). 



Fig. 3. Local configurations of a 26-simple point with $\mathcal{N}_{6}(p) \cap X=\emptyset$

Case 2: $2 \geq \operatorname{card}\left(\mathcal{N}_{6}(p) \cap X\right) \geq 1$. Since $\mathcal{N}(p)$ is 26-simple, $p$ is, up to symmetries, as in one of the configurations of Figure 2, where the gray points may or may not be in $X$. The gray points may depend of their neighbors (for example, in the second case, if nbw is black, then $n w$ must be also black). 
In all cases, we subdivide $p$ in up to 5 parts as in Figure 2 (in some cases, to better see the subdivision, one of the subsets is shown transparent) and we define $f$ inducing $F$ such that the image of the smallest parts are always the blue or the gray point they are "oriented to" (i.e. that for which this region is the closest). Each of these parts is adjacent to a bigger part which goes to a blue o gray point adjacent to the former, and so on. If any of the gray points were not in $X$ its part of the subdivision would be absorbed by its neighboring part.

Case 3: $\operatorname{card}\left(\mathcal{N}_{6}(p) \cap X\right)=0$. If $\mathcal{N}_{18}(p) \cap X=\emptyset$, then $\mathcal{N}(p) \cap X$ consists on just one corner of $\mathcal{N}(p), q$, and we define $F(p)=q$, In the rest of the cases $\mathcal{N}_{18}(p) \cap X \neq \emptyset$. All possible configurations are shown in Figure 3.

The seven first cases satisfy that there is at least a white point $q$ 6-adjacent to $p$ with $\operatorname{card}\left(\mathcal{N}_{6}(q) \cap \mathcal{N}(p) \cap\left(\mathbb{Z}^{3} \backslash X\right)\right) \geq 3$. The four last cases satisfy that $\operatorname{card}\left(\mathcal{N}_{6}(q) \cap \mathcal{N}(p) \cap\left(\mathbb{Z}^{3} \backslash X\right)\right) \leq 2$ for all $q$ 6-adjacent to $p$.

Note that, as in case 2 , the gray points may depend of their neighbors (for example, in the first case, if $n b w$ is black, then $b w$ must be also black). Moreover, not all combinations of gray points are possible (for example, in the second case, if $f e$ is white, all the gray points must be also white).

In all cases, we subdivide $p$ in up to 7 parts as in Figure 3 (in some cases, to better see the subdivision, one of the subsets is shown transparent), and we define $f$ inducing $F$ following the same rule as in case 2 (now, when two points can be chosen as the next one, only one of them allows to define the image of all the remaining subsets).

In Figure 4 we show the steps to construct the subdivision in one of the three last cases (the ninth) which are more involved.
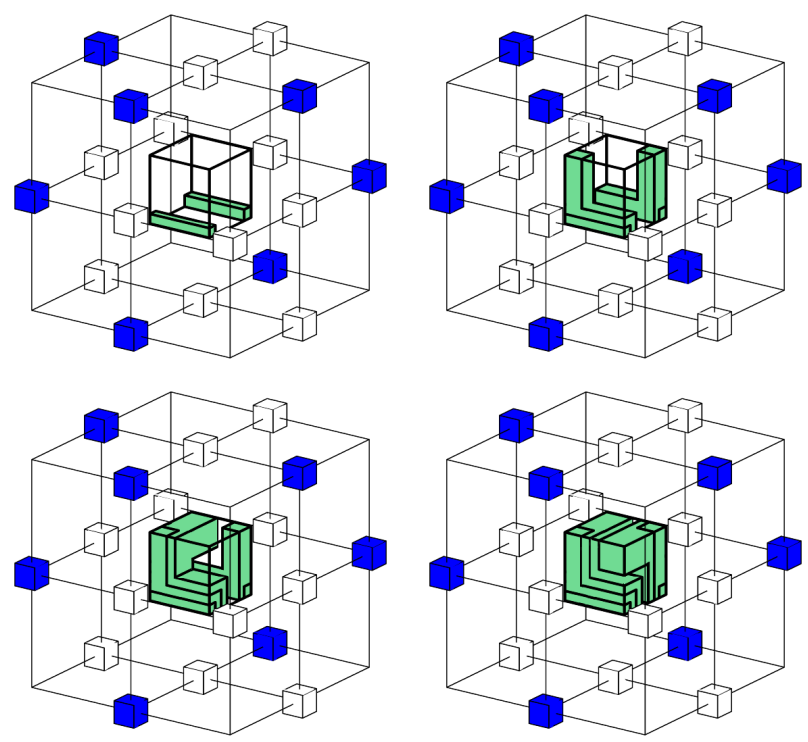

Fig. 4. Construction of the subdivision of $p$ in one case 
Remark 1. The previous theorem guarantees that, if $p \in X \subset \mathbb{Z}^{3}$ is a 26-simple point, then there exists an $(\mathcal{N}, 26)$-retraction $F: X \longrightarrow X \backslash\{p\}$.

Moreover, the multivalued $(\mathcal{N}, 26)$-retraction $F: X \longrightarrow X \backslash\{p\}$ constructed in the proof of the theorem is given by $F(x)=\{x\}$ if $x \neq p$ and

$$
\begin{aligned}
F(p)=\left(\mathcal{N}_{6}(p) \cap X\right) & \cup\left\{q \in \mathcal{N}_{18}^{*}(p) \cap X \mid \mathcal{N}_{6}(p) \cap \mathcal{N}_{6}(q) \cap X=\emptyset\right\} \\
& \cup\left\{q \in \mathcal{N}_{26}^{*}(p) \cap X \mid \mathcal{N}_{18}(p) \cap \mathcal{N}_{18}(q) \cap X=\emptyset\right\},
\end{aligned}
$$

where $\mathcal{N}_{18}^{*}(p)=\mathcal{N}_{18}(p) \backslash \mathcal{N}_{6}(p)$ and $\mathcal{N}_{26}^{*}(p)=\mathcal{N}_{26}(p) \backslash \mathcal{N}_{18}(p)$. Note that, since $p$ is 26-simple, the third term in the union is nonempty if and only if $\mathcal{N}(p) \cap X$ is just one corner of $\mathcal{N}(p)$.

Analogously, it can be easily seen, using the techniques of [5,6], that if $X \subset \mathbb{Z}^{2}$ and $p \in X$, then the multivalued function $F: X \longrightarrow X \backslash\{p\}$ given by $F(x)=\{x\}$ if $x \neq p$ and

$$
F(p)=\left(\mathcal{N}_{4}(p) \cap X\right) \cup\left\{q \in \mathcal{N}_{8}^{*}(p) \cap X \mid \mathcal{N}_{4}(p) \cap \mathcal{N}_{4}(q) \cap X=\emptyset\right\} .
$$

is a multivalued $(\mathcal{N}, 8)$-retraction if and only if $p$ is an 8 -simple point.

\section{Conclusions and Future Work}

We have shown that if $p$ is a 26-simple point of $X \subset \mathbb{Z}^{3}$ then there exists a multivalued $(\mathcal{N}, 26)$-retraction $F: X \longrightarrow X \backslash\{p\}$.

Conversely, it can be proved, in a similar way as for Theorem 2, that, if there exists a multivalued $(\mathcal{N}, 26)$-retraction $F: X \longrightarrow X \backslash\{p\}$, then $p$ is a 26 -boundary point of $X$ such that the number of 26-connected components of $\mathcal{N}(p) \cap X$ which are 26 -adjacent to $p$ is equal to 1 , and there exists at least one 6-connected components of $\mathcal{N}_{18}(p) \cap\left(\mathbb{Z}^{3} \backslash X\right)$ 6-adjacent to $p$ (i.e $p$ is a 26-boundary point).

Moreover, in all the configurations considered in the proof of Theorem 3 , if we 6 -disconnect the "white" points, a multivalued $(\mathcal{N}, 26)$-retraction $F: X \longrightarrow$ $X \backslash\{p\}$ not longer exists. In general, if the deletion of $p$ creates a hole in $X$, a multivalued $(\mathcal{N}, 26)$-retraction $F: X \longrightarrow X \backslash\{p\}$ does not exist. Therefore, the following holds: " $p$ is a 26-simple point of $X \subset \mathbb{Z}^{3}$ if and only if there exists a multivalued $(\mathcal{N}, 26)$-retraction $F: X \longrightarrow X \backslash\{p\}$ ". We will publish the proof of this result in a forthcoming paper.

\section{References}

1. Bertrand, G., Mandalain, G.: A new characterization of three-dimensional simple points. Pattern Recognition Letters 15, 169-175 (1994)

2. Boxer, L.: Digitally continuous functions. Pattern Recognition Letters 15, 833-839 (1994)

3. Boxer, L.: A Classical Construction for the Digital Fundamental Group. Journal of Mathematical Imaging and Vision 10, 51-62 (1999) 
4. Boxer, L.: Properties of Digital Homotopy. Journal of Mathematical Imaging and Vision 22, 19-26 (2005)

5. Escribano, C., Giraldo, A., Sastre, M.A.: Digitally Continuous Multivalued Functions. In: Coeurjolly, D., Sivignon, I., Tougne, L., Dupont, F. (eds.) DGCI 2008. LNCS, vol. 4992, pp. 81-92. Springer, Heidelberg (2008)

6. Escribano, C., Giraldo, A., Sastre, M.A.: Digitally continuous multivalued functions, morphological operations and thinning algorithms. Journal of Mathematical Imaging and Vision 42(1), 76-91 (2012)

7. Fourey, S., Malgouyres, M.: A concise characterization of 3D simple points. Discrete Applied Mathematics 125, 59-80 (2003)

8. Khalimsky, E.: Topological structures in computer science. Journal of Applied Mathematics and Simulation 1, 25-40 (1987)

9. Klette, R., Rosenfeld, A.: Digital Geometry. Elsevier (2004)

10. Kong, T.Y.: A digital fundamental group. Comp. Graphics 13, 159-166 (1989)

11. Kong, T.Y., Rosenfeld, A.: Digital Topology: Introduction and survey. Computer Vision, Graphic and Image Processing 48, 357-393 (1989)

12. Kong, T.Y., Rosenfeld, A. (eds.): Topological algorithms for digital image processing. Elsevier (1996)

13. Kovalevsky, V.: A new concept for digital geometry. In: Ying-Lie, O., et al. (eds.) Shape in Picture. Proc. of the NATO Advanced Research Workshop, Driebergen, The Netherlands (1992); Computer and Systems Sciences 126. Springer (1994)

14. Rosenfeld, A.: Digital topology. The American Mathematical Monthly 86(8), 621-630 (1979)

15. Rosenfeld, A.: Continuous functions in digital pictures. Pattern Recognition Letters 4, 177-184 (1986) 